\title{
Disparities and Poor Outcomes for Patients Diagnosed with Lung Cancer Through the Emergency Department
}

$1^{\text {st }}$ Nathaniel Blucker ${ }^{1}, 2^{\text {nd }}$ Nicholas Pettit ${ }^{2}$

${ }^{1}$ Indiana University School of Medicine, ${ }^{2}$ Indiana University School of Medicine, Department of Emergency Medicine

\section{Background and Hypothesis:}

Cancer is currently the second leading cause of death in the United States, with lung cancer being the leading cause of cancer death. Approximately $40 \%$ of patients with lung cancer are diagnosed emergently, many at advanced stages, and occur despite the existence of lung cancer screening. However, local trends among Eskenazi Health patients are unknown. We hypothesize that individuals diagnosed with lung cancer through emergency presentations at Eskenazi Health will experience greater negative outcomes than individuals who are diagnosed through elective routes.

\section{Experimental Design of Project Methods:}

This is a retrospective chart review utilizing the Eskenazi Health electronic medical health records system. Patients with an ICD-coded lung cancer diagnosis within the five-year period between 2016 and 2021 were analyzed. We compared characteristics, health care utilization, and outcomes for adult patients who had a diagnosis of lung cancer. Our primary outcome was the number of patients diagnosed with lung cancer emergently.

\section{Results:}

Thus far, $74 \%$ of the cases viewed have been considered emergent diagnoses of lung cancer. Of the total cases, $54 \%$ of emergent diagnoses and $32 \%$ of non-emergent are stage IV. Mortality rates of both stage IV groups respectively are $74 \%$ and $62.5 \%$. Overall mortality rates were $56.6 \%$, and $45.9 \%$ for emergent and non-emergent diagnoses respectively. Amongst the diagnoses viewed, $93.8 \%$ failed to occur due to lung cancer screening. Rates of screening amongst the emergent group were lower at $6.6 \%$, as opposed to non-emergent cases at $24 \%$.

\section{Conclusion and Potential Impact:}

A significant portion of lung cancer diagnoses at Eskenazi Health are taking place due to presentation through emergent routes. The stage of these diagnoses suggests possible missed opportunities for earlier diagnosis. Additionally, a large part of the patient population currently qualifies for lung cancer screening despite low rates of screening being performed. Future work should emphasize reducing the number of patients emergently diagnosed with lung cancer. 